\title{
Wavepath eikonal traveltime inversion: Theory
}

\author{
Gerard T. Schuster* and Aksel Quintus-Bosz*
}

\section{ABSTRACT}

We present a general formula for the back projection of traveltime residuals in traveltime tomography. For special choices of an arbitrary weighting factor this formula reduces to the asymptotic back-projection term in ray-tracing tomography (RT), the WoodwardRocca method, wavepath eikonal traveltime inversion (WET), and wave-equation traveltime inversion (WT). This unification provides for an understanding of the differences and similarities among these traveltime tomography methods.

The special case of the WET formula leads to a computationally efficient inversion scheme in the space-time domain that is, in principle, almost as effective as WT inversion yet is an order of magnitude faster. It also leads to an analytic formula for the fast computation of wavepaths. Unlike ray-tracing tomography, WET partially accounts for band-limited source and shadow effects in the data. Several numerical tests of the WET method are used to illustrate its properties.

\section{INTRODUCTION}

Woodward and Rocca (1988) used the imaginary part of the Rytov inversion formula (Keller, 1969) to invert for the velocity field from traveltime data. Velocities are reconstructed by back-projecting phase residuals along wavepaths associated with source-receiver pairs. Woodward (1989) showed that a wavepath, similar to a "fat" ray, defines the propagation path of band-limited seismic energy from the source point to the receiver point. Its chief merit is that it accounts for some phenomena not modeled by simple ray tracing, e.g., shadow zone and multipathing effects. The penalty, however, is that it is computationally costly compared to ray tracing, requiring a finite-difference solution to the Helmholtz equation at each frequency for every source position and every receiver position. For example, if there are $\mathrm{S}$ sources, $\mathrm{R}$ receivers, and $F$ frequencies, then the Helmholtz equation must be solved $F^{*}(S+R)$ number of times at each iteration (Pratt and Worthington, 1990). For the significant scattering case, the Woodward and Rocca method (WR) inverts the phase of the entire seismogram. Woodward (1992) states "Because it is monochromatic, modeled events cannot be simply windowed from unmodeled events in time: all of the signal and noise must be dealt with simultaneously."

Harlan (1990) used the wavepath concept of Woodward and Rocca (1988) and developed a traveltime tomography method by constructing wavepaths from eikonal equation traveltimes. The traveltime residuals are back projected along these wavepaths to update the slowness field.

Luo and Schuster (1990, 1991a, and 1991b) developed a wave-equation traveltime inversion (WT) method in the space-time domain. Because of its space-time formulation, the first arrivals can be isolated and inverted independent of later arrivals; this gives rise to a robust inversion for complicated velocity models. Velocities are reconstructed by reverse time migration of the weighted observed seismograms, where the seismogram weight is the traveltime residual (i.e., difference between the computed and observed first arrival traveltimes) associated with that seismogram. The calculated transmission traveltimes are those from the synthetic seismograms computed by a finite-difference solution to the wave equation. Luo (1991) recently showed that the WT method can be interpreted as back-projecting traveltime residuals along wavepaths, similar to the Woodward and Rocca (1988) method. The problem with the WT method is similar to that of the Woodward and Rocca method, i.e., it can require more than an order of magnitude more CPU time than that of simple ray-tracing tomography (RT). For example, if there are $\mathrm{S}$ sources and $R$ receivers, then $\mathrm{S}$ reversetime common-shot point gather (CSP) migrations must be carried out for each iteration, where a CSP gather contains $R$ observed seismograms.

To overcome the CPU expense of both the WR and WT methods, this paper presents a traveltime inversion method that accounts for some wavepath effects, yet its computational costs are comparable to that of ray-tracing tomogra-

Manuscript received by the Editor December 6, 1991; revised manuscript received December 30, 1992.

Department of Geology and Geophysics, University of Utah, Salt Lake City, UT 84112.

(C) 1993 Society of Exploration Geophysicists. All rights reserved. 
phy. We call this method wavepath eikonal traveltime (WET) inversion because it computes wavepaths by using finite-difference solutions to the eikonal equation (Schuster, 1991). Unlike the WT or Woodward-Rocca methods, it is considered a high-frequency method because it uses solutions to the eikonal equation. Unlike ray-tracing tomography, however, WET partially accounts for the band-limited effects of the source wavelet and diffraction effects. Figure 1 roughly illustrates the CPU costs versus frequency for the WT, WET and RT methods.

The first section in this paper presents the derivation of a general formula for the back projection of phase or traveltime residuals. The second section plugs the asymptotic Green's function into the general back projection formula to yield traveltime back-projection operators that link the RT, WR, WT, and WET methods to each other. The third section illustrates the WET algorithm by numerical examples and the last section presents the conclusions.

\section{GENERAL ASYMPTOTIC BACK-PROJECTION FORMULA}

We will now derive a general formula for back projecting traveltime residuals, which can be used to invert for the slowness field $\mathrm{s}(\mathrm{x})$ in an acoustic medium. It will be assumed that the seismic data are recorded in a seismic cross-well experiment with vertical source and receiver wells. The data are collected in the space-time domain and the first arrivals in the space-time seismograms are windowed and Fourier transformed in time to obtain the pressure field $\tilde{p}_{\text {cal }}\left(\mathbf{x}_{r}, \omega \mid \mathbf{x}_{s}, 0\right)$ measured at $\mathbf{x}_{r}$, where the angular frequency is denoted by $\omega$. The energy originates from a point source at $\mathbf{x}_{s}$, and the first arrival traveltimes are assumed to be picked from the peak amplitude of a shifted zero-phase vibroseis wavelet.

The following steps lead to the general formula for the back projection of traveltime or phase residuals.

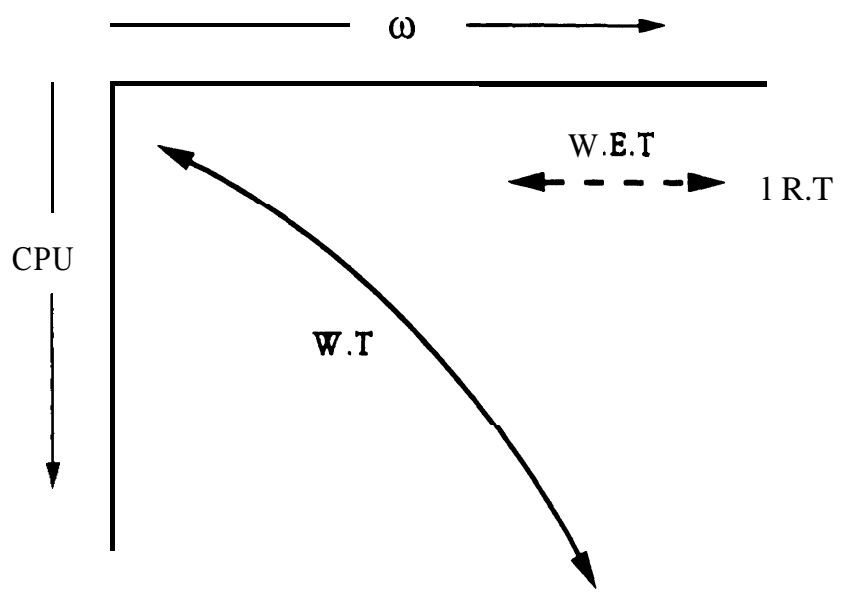

FIG. 1. CPU costs versus frequency for WET, WT, and RT methods. The RT method is valid at very high frequencies because it assumes that the path of energy propagation is along an infinitesimally thin ray tube. The WET method accounts for a greater range of high frequencies because it models wave propagation along wavepaths; i.e., when the source bandwidth has finite width. However, it is invalid when scattering effects are dominant or when the characteristic scale of the medium is about the same as or smaller than the dominant source wavelength. The WT method is valid over all frequencies.
1) Form a phase misfit function $\varepsilon$

$$
\varepsilon=\frac{1}{2} \sum_{s} \sum_{r} \sum_{\omega} \tilde{R}_{r s}(\omega) \Delta \phi\left(\mathbf{x}_{r}, \mathbf{x}_{s}, \omega\right)^{2},
$$

where the summations are over the receiver $r$ and source $s$ indices and over the discrete source frequen${ }^{\text {cies }} \omega ; \Delta \phi\left(\mathbf{x}_{r}, \mathbf{x}_{s}, \omega\right)=\phi\left(\mathbf{x}_{r}, \mathbf{x}_{s}, \omega\right)_{c a l}-\phi\left(\mathbf{x}_{r}, \mathbf{x}_{s}\right.$, $\omega)_{o b s}$ is the phase residual, or difference between the calculated $\phi\left(\mathbf{x}_{r}, \mathbf{x}_{s}, \omega\right)_{c a l}$ and observed $\phi\left(\mathbf{x}_{r}, \mathbf{x}_{s}\right.$, $\omega)_{o b s}$ phases of the first arrivals at a single frequency. The associated observed $\tilde{p}_{\text {obs }}\left(\mathbf{x}_{r}, \omega \mid \mathbf{x}_{s}, 0\right)$ and calculated $\tilde{p}_{c a l}\left(\mathbf{x}_{r}, \omega \mid \mathbf{x}_{s}, 0\right)$ pressure fields satisfy the Helmholtz equation. An arbitrary weighting factor is denoted as $\tilde{R}_{r s}(\omega)$, and if it is set equal to the magnitude spectrum of the source wavelet then it accounts for the reliability (akin to a probability function) of the phase measurements as a function of frequency. Its chief effect will be to smooth the gradient (or reconstructed slowness field) in a way that is consistent with the source spectrum and the path of propagation. This is more physically appealing than ad hoc smoothing methods (e.g., Gersztenkorn and Scales, 1988).

2) Find the gradient $y(x)$ of the phase misfit function with respect to the slowness parameters

$$
\begin{aligned}
\gamma(\mathbf{x})= & \frac{\partial \varepsilon}{\partial s(\mathbf{x})}=\sum_{s} \sum_{r} \sum_{\omega} \widetilde{R}_{r s}(\omega) \frac{\partial \phi_{c a l}\left(\mathbf{x}_{r}, \mathbf{x}_{s}, \omega\right)}{\partial s(\mathbf{x})} \\
& \times \Delta \phi\left(\mathbf{x}_{r}, \mathbf{x}_{s}, \omega\right),
\end{aligned}
$$

so that the slowness can be reconstructed by a, say steepest descent, update formula

$$
s(\mathbf{x})^{(k+1)}=s(\mathbf{x})^{(k)}-\alpha^{(k)} \gamma(\mathbf{x})
$$

where the $k$ index denotes the kth iteration and $\alpha^{(k)}$ is the step length.

3) Compute $\partial \phi_{c a l}\left(\mathbf{x}_{r}, \mathbf{x}_{s}, \omega\right) / \partial s(\mathbf{x})$ by using the definition of phase $\phi_{c a l}\left(\mathbf{x}_{r}, \mathbf{x}_{s}, \omega\right)=\operatorname{Im}\left[\ln \tilde{p}_{c a l}\left(\mathbf{x}_{r}, \omega \mid \mathbf{x}_{s}, 0\right)\right]$ and taking the derivative of $\phi_{c a l}\left(\mathbf{x}_{r}, \mathbf{x}_{s}, \omega\right)$ with respect to $\mathrm{s}(\mathrm{x})$ to get

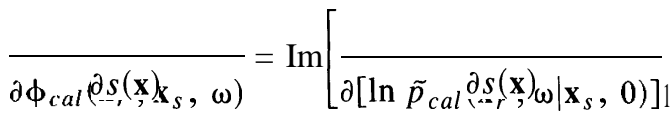

$$
\begin{aligned}
& =\frac{\mathrm{I} \frac{\partial \tilde{p}_{c a l}\left(\mathbf{x}_{r}, \omega \mid \mathbf{x}_{s}, 0\right)}{\partial s(\mathbf{x})} \mid}{\left|\tilde{p}_{c a l}\left(\mathbf{x}_{r}, \omega \mid \mathbf{x}_{s}, 0\right)\right|} \sin \left(\phi^{\prime}-\phi_{c a l}\right) .
\end{aligned}
$$

Here, the calculated phase is denoted by $\phi_{\text {cal }}=$ $\phi_{\text {cal }}\left(\mathbf{x}_{r}, \mathbf{x}_{s}, \omega\right)$ and $\phi^{\prime}=\phi\left(\mathbf{x}_{r}, \mathbf{x}_{s}, \mathbf{x}, \omega\right)$ is defined to be the phase of $\partial \tilde{p}_{c a l}\left(\mathbf{x}_{r}, \omega \mid \mathbf{x}_{s}, 0\right) / \partial s(\mathbf{x})$. It can be shown (Tarantola, 1987) that the Frechet derivative of the pressure field is given as

$$
\begin{aligned}
& \frac{\partial \tilde{p}_{c a l}\left(\mathbf{x}_{r}, \omega \mid \mathbf{x}_{s}, 0\right)}{\operatorname{as}(\mathrm{x})}- 2 s(\mathbf{x}) \omega^{2} \tilde{p}_{c a l}\left(\mathbf{x}, \omega \mid \mathbf{x}_{s}, 0\right) \\
& \times \tilde{g}\left(\mathbf{x}_{r}, \omega \mid \mathbf{x}, 0\right),
\end{aligned}
$$

where $\tilde{g}\left(\mathbf{x}_{r}, \omega \mid \mathbf{x}, 0\right)$ is the three-dimensional (3-D inhomogeneous Helmholtz Green's function for al 
impulse source at $\mathrm{x}$ and an observer at $\mathrm{x}$, . Substituting equation (5) into (4), and substituting the resulting expression for $\partial \phi_{c a l}\left(\mathbf{x}_{r}, \mathbf{x}_{s}, \omega\right) / \partial s(\mathbf{x})$ into equation (2) yields

$$
\begin{aligned}
\gamma(\mathbf{x})= & 2 s(\mathbf{x}) \sum_{s} \sum_{r} \sum_{\omega} \omega^{2} \Delta \phi\left(\mathbf{x}_{r}, \mathbf{x}_{s}, \omega\right) \tilde{R}_{r s}(\omega) \\
& X \frac{\left|\tilde{p}_{c a l}\left(\mathbf{x}, \omega \mid \mathbf{x}_{\mathbf{s}}, \mathbf{0}\right)\right|\left|\tilde{\mathbf{g}}\left(\mathbf{x}, \omega \mid \mathbf{x}_{r}, 0\right)\right|}{\mathrm{I} \tilde{p}_{c a l}\left(\mathbf{x}_{r}, \omega \mid \mathbf{x}_{s}, 0\right) \mid} \\
& \times \sin \left(\phi^{\prime}-\phi_{c a l}\right),
\end{aligned}
$$

where $\phi^{\prime}=\phi_{g}+\phi_{p}$, and the phase of the Green's function and the phase of the calculated pressure are given by, respectively, $\phi_{g}=\phi_{g}\left(\mathbf{x}, \mathbf{x}_{r}, \omega\right)$ and $\phi_{p}=$ $\phi_{p}\left(\mathbf{x}, \mathbf{x}_{s}, \omega\right)$.

4) The asymptotic gradient for a single source at $\mathbf{x}_{s}$ and receiver at $\mathbf{x}_{r}$ can be found by linearizing phase with respect to the first-arrival traveltime (i.e., set $\Delta \phi\left(\mathbf{x}_{r}\right.$, $\left.\left.\mathbf{x}_{s}, \omega\right)=\omega\left(\tau_{r s}-\tau_{r s}^{o b s}\right)=\omega \Delta \tau\right)$, and substituting the geometrical optics Green's function (Miller et al., 1987)

$$
\tilde{g}\left(\mathbf{x}, \omega \mid \mathbf{x}_{r}, 0\right)=\tilde{p}\left(\mathbf{x}, \omega \mid \mathbf{x}_{r}, 0\right)=\frac{e^{l \omega \tau} x r}{A_{x r}},
$$

into equation (6) to get

$$
\begin{aligned}
\gamma(\mathbf{x})= & \frac{2 s(\mathbf{x}) A, \Delta \tau}{A_{x r} A_{x s}} \\
& \cdot \int_{-\infty}^{\infty} \omega^{3} \widetilde{R}_{r s}(\omega) \sin \left(\phi^{\prime}-\phi_{c a l}\right) d \omega .
\end{aligned}
$$

Substituting $\phi^{\prime}-\phi_{c a l}=\omega\left(\tau_{x s}+\tau_{x r}-\tau_{r s}\right)$ into the argument of the sine we have

$$
\begin{aligned}
\gamma(\mathbf{x})= & \frac{2 s(\mathbf{x}) A, \Delta \tau}{A_{x r} A_{x s}} 1_{\mathrm{I}-\infty}^{\infty} \omega^{3} \widetilde{R}_{r s}(\omega) \\
& \times \sin \left(\omega\left[\tau_{x s}+\tau_{x r}-\tau_{r s}\right]\right) d o .
\end{aligned}
$$

Here, $\tau_{x x^{\prime}}$ is defined to be the first-arrival traveltime solution to the eikonal equation for a source at $\mathbf{x}^{\prime}$ and receiver at $\mathrm{x}$ in a slowness distribution $\mathrm{s}(\mathrm{x})$; and $A_{x x^{\prime}}$ is the associated geometrical spreading term of the first arrival whose reciprocal satisfies the transport equation. The linearization of phase with respect to frequency means that we ignore scattering effects in the data.

\section{UNIFICATION OF TOMOGRAPHY ALGORITHMS}

Examining special cases of the weighting factor $\widetilde{R}_{r s}(\mathrm{w})$ in equation (8) illuminates the physical meaning of the asymptotic gradient. These special cases also lead to the asymptotic back-projection formulas for ray-tracing tomography, the Woodward and Rocca (1988) method, wave-equation traveltime inversion (Luo and Schuster, 1991a), and wavepath eikonal traveltime inversion (Schuster, 1991; Quintus-Bosz, 1992). The weighting factors are summarized in Table 1.

Case 1: Inhomogeneous medium and impulse source

Assuming an impulsive point source [i.e., $\widetilde{R}_{r s}(\mathrm{w})=$ $1 /(2 \pi)$ ] and an inhomogeneous slowness distribution $\mathrm{s}(\mathrm{x})$ between source and receiver wells, equation (8) becomes

$$
\gamma(\mathbf{x})=\frac{2 s(\mathbf{x}) A,, 1 \Delta \tau}{A_{x r} A_{\mathrm{XS}}} \delta^{\prime \prime \prime}\left(\tau_{x s}+\tau_{x r}-\tau_{r s}\right) .
$$

Here, triple prime indicates triple differentiation with respect to the argument. Equation (9) says that the support of $y(x)$ is along the first-arrival raypath in the medium, and therefore the model velocities are adjusted only along that Fermat raypath.

Case 2: Inhomogeneous medium+ wideband source + weighting $=$ ray tomography

Since velocities primarily influence the phase delays, it might seem reasonable to eliminate the amplitude factors and retain just the phase factors in equation (8). This can be done by setting

$$
\tilde{R}_{r s}(\omega)=\tilde{W}(\omega) \frac{A_{x r} A_{x s}}{2 \omega^{3} s(\mathbf{x}) A_{r s}} H,
$$

in equation (8), where $H=1 /(\pi \omega)^{*}$ is the convolutional Hilbert transform operator in $\omega$ and $\widetilde{W}(\omega)$ is the wavelet magnitude spectrum. Substituting equation (10a) into equation (8) yields

$$
\gamma(\mathbf{x})=W\left(\tau_{x s}+\tau_{x r}-\tau_{r s}\right) \Delta \tau,
$$

where $W(t)$ is the inverse Fourier transform of $\widetilde{W}(\omega)$. Dividing out the geometric spreading terms is equivalent to the preconditioning step in Beydoun and Mendes (1989), dividing by the cube of the angular frequency is similar to applying a low-pass filter (Gersztenkorn and Scales, 1988), and the application of the Hilbert transform is somewhat

Table 1. Tomography algorithms and weighting factor $\widetilde{\boldsymbol{R}}_{\boldsymbol{r} \boldsymbol{s}}(\boldsymbol{\omega})$ in Equation (8).

\begin{tabular}{lc}
\hline Tomography Method & $\widetilde{R}_{r s}(\omega)$ weight in equation (8) \\
\cline { 2 - 2 } Fat RT equation (10b) & $\widetilde{W}(\omega) A_{x r} A_{x s} H /\left(2 \omega^{3} s(\mathbf{x}) A_{r s}\right)$ \\
Thin RT (equation (10c) & $A_{x r} A_{x s} H /\left(2 \omega^{3} s(\mathbf{x}) A_{r s}\right)$ \\
WET equation (11c) & $\widetilde{W}(\omega)$ \\
WR equation (12) & $-1 / \omega^{2}$ \\
WT equation (13) & $2 \operatorname{Im}\left(i \tilde{p}\left(\mathbf{x}_{\mathbf{r}}, \omega \mid \mathbf{x}_{\mathbf{s}}, 0\right)_{o b s} e^{i \omega \tau_{o b s}}\right) / \ddot{p}\left(\mathbf{x}_{\mathbf{r}}, \tau_{r s}^{o b s} \mid \mathbf{x}_{\mathbf{s}}, 0\right)_{o b s}$ \\
\hline
\end{tabular}


related to a frequency domain quelling operation (Meyerholtz et al., 1989) that transforms the sine in equation (8) into a cosine.

For $\widetilde{W}(\omega)=1 /(2 \pi)$ and a slowness parameterization into cells of constant slowness, $\mathrm{W}(\mathrm{t})$ becomes

$$
\gamma(\mathbf{x})=\delta\left(\tau_{x s}+\tau_{x r}-\tau_{r s}\right) \Delta t,
$$

so that the gradient in equation (10b) is proportional to that of ray-tracing tomography, namely the raypath length in a cell multiplied by the traveltime residual. If $\mathrm{W}(\mathrm{t})$ is a Gaussian function then the infinitesimally thin ray widens into a "fat" ray (Harlan, 1990). The concept of a fat ray was recently described as Fresnel volume ray tracing by Cerveny and Soares (1992), although equation (10b) takes into account both the correct Fresnel zone and the wavelet shape. Tracing rays by setting the argument of $W$ in equation (10b) to zero was also used by Matsuoka and Ezaka (1992).

Case 3: Inhomogeneous medium and narrow-band source $=$ WET

This is the same as Case 1 except for a flat narrow-band source with a center frequency of $\omega_{c}$ and a bandwidth of $2 \omega_{0}$. Setting $R,,(0)=1 / 2$ in equation (8) and replacing the integration by

$$
\int_{-\omega_{c}-\omega_{0}}^{-\omega_{c}+\omega_{0}} d \omega+\int_{\omega_{c}-\omega_{0}}^{\omega_{c}+\omega_{0}} d o
$$

we get

$$
\begin{aligned}
\gamma(\mathbf{x})= & \frac{4 \omega_{0} s(\mathbf{x}) A, \text { AT }}{A_{x r} \cdot A_{x s}} \\
& \times\left[\operatorname{sinc}\left(\frac{\omega_{0}}{\pi} \sum \tau\right) \cos \left(\omega_{c} \sum \tau\right)\right]^{\prime \prime \prime},
\end{aligned}
$$

where $\sum \tau=\tau_{x s}+\tau_{x r}-\tau_{r s}$. Note that the central frequency $\omega_{c}$ determines the zero crossings of the cosine function, and the bandwidth frequency $\omega_{0}$ determines the zero crossings of the modulating sinc function. The formula verifies Woodward's (1992) conclusion that the wavepath's zero crossings are governed by the central source frequency.

If $\mathrm{o}, \approx 0$ then the gradient becomes

$$
\begin{aligned}
\gamma(\mathbf{x})= & \frac{4 \omega_{0} s(\mathbf{x}) A, \mathrm{AT}}{A \mathrm{Xi}_{\mathrm{i}} * \mathrm{~A},} \\
& \times \operatorname{sinc}^{\prime \prime \prime}\left(\frac{\omega_{0}}{\pi}\left(\tau_{x s}+\tau_{x r}-\tau_{r s}\right)\right) 1
\end{aligned}
$$

Equation (11b) is similar to equation (9) except that the delta function is replaced by a weighted sinc function. It associates the band-limited gradient function with a sinc function that carries phase information modulated by the geometrical spreading terms. The contours of the sinc function define surfaces which are coincident with the aplanatic surfaces of equal transmission traveltime. Equation $(1 \mathrm{lb})$ says that the traveltime residuals are back-projected into the medium along "sincpaths" (rather than raypaths) and weighted along these surfaces of constant phase or traveltime. This is consistent with the concept of back propagating traveltime residuals along "wavepaths" introduced by Waodward and Rocca (1988) and later developed by Luo (1991).

If $\widetilde{R}_{r s}(\omega)$ in equation (8) is replaced by the magnitude spectrum of say, a Ricker wavelet, then the triple derivative sinc function in equation $(1 \mathrm{lb})$ is replaced by the timedomain Ricker wavelet $W^{\prime \prime \prime}\left(\tau_{x s}+\tau_{x r}-\tau_{r s}\right) / 2 \omega_{0}$, i.e.,

$$
\gamma(\mathbf{x})=\frac{2 s(\mathbf{x}) A, \mathrm{AT}}{A_{x r} \cdot A_{\mathrm{xS}}} W^{\prime \prime \prime}\left(\tau_{x s}+\tau_{x r}-\tau_{r s}\right),
$$

which is the asymptotic gradient for the wavepath eikonal traveltime inversion or WET method (Schuster, 1991; Quintus-Bosz, 1992). Unlike the sinc function in equation $(1 \mathrm{lb})$, the wavepath associated with equation $(1 \mathrm{lc})$ is shaped by the magnitude spectrum of the source wavelet. This is physically consistent with the actual path of wave propagation for a shifted zero phase wavelet.

\section{Case 4: Asymptotic Woodward-Rocca gradient method}

If $\widetilde{R}_{r s}(\omega)$ is set equal to $-1 / \omega^{2}$ and the frequency summation replaced by an integration over finite limits, then equation (8) reduces to the asymptotic Woodward-Rocca backprojection formula (see the Appendix), i.e.,

$\gamma(\mathbf{x})=\frac{2 s(\mathbf{x}) A, \text { AT }}{A_{x s} A_{x r}} \operatorname{sinc}^{\prime}\left[\omega_{0}\left(\tau_{x s}+\tau_{x r}-\tau_{r s}\right) / \pi\right]$.

Some salient differences between this gradient and the WET gradient in equation $(1 \mathrm{lc}$ ) are that equation (12) contains no information about the source wavelet signature $W(t)$ and that it is a single derivative rather than a triple derivative of the sinc function. Thus, equation (12) is a low-pass filtered version of a special case of WET in equation $(1 \mathrm{lb})$. Another difference is that the linearized WR gradient is computed by first calculating the wavepaths at each frequency (by solving the Helmholtz equation), and then numerically summing each wavepath to get the multifrequency wavepath gradient. WET, on the other hand, avoids this costly wavepath computation at each frequency and simply evaluates the formula in equation (1 lc).

Case 5: Asymptotic wave equation traveltime inversion

If $\widetilde{R}_{r s}(03)$ in equation (8) is set equal to the weighted spectrum of the first arrival (see the Appendix), i.e.,

$$
\tilde{R}_{r s}(\omega)=\frac{2 \operatorname{Im}\left(i \bar{p}\left(\mathbf{x}_{r} \omega \mid \mathbf{x}_{s}, 0\right)_{o b s} e^{i \omega \tau} \tau_{r s}^{a b s}\right)}{\ddot{p}\left(x_{r}, \tau_{r s}^{o b s} \mid \mathbf{x}_{s}, 0\right)_{o b s}},
$$

then this reduces to the asymptotic gradient for the WT method [see equation (A-8)] of Luo and Schuster (1991a). Here, a double dot indicates double time differentiation and $p\left(\mathbf{x}_{r}, t \mid \mathbf{x}_{s}, 0\right)$ is the space-time pressure seismogram for a source at $\mathbf{x}_{s}$ and receiver at $\mathbf{x}_{r}$. Note that the WT gradient uses the actual first arrival windowed from the observed seismogram, not just the source wavelet. Unlike the WET or RT methods, this partially takes into account the effects of scattering and source radiation pattern in the data.

\section{NUMERICAL WET ALGORITHM}

Equation $(1 \mathrm{lc})$ suggests a computationally fast methodology for inverting $\mathrm{s}(\mathrm{x})$ from traveltimes computed by a 
finite-difference solution to the eikonal equation (Qin et al., 1992); it also suggests a fast means for the calculation of wavepaths associated with the eikonal traveltimes. The algorithm for wavepath eikonal traveltime inversion (WET) is:

1) Pick the first-arrival traveltimes $\tau_{r s}^{o b s}$ from the seismograms.

2) An initial slowness model is proposed and the eikonal equation is efficiently solved by a finite-difference method (Qin et al., 1992) to get $\tau_{x s}$ and $\tau_{x r}$. The traveltime residual $\Delta \tau=\tau_{r s}-\tau_{r s}^{o b s}$ is computed by subtracting the observed first-arrival traveltimes from the finite-difference traveltimes.

$3)$ The source weighting function in equation $(1 \mathrm{lc})$ is evaluated at all points within the medium to give $\mathrm{y}(\mathrm{x})$. In practice, we also include summations over source and receiver positions to account for the multiple sources and receivers in the experiment. The geometrical spreading terms can be computed by a finitedifference computation (Pusey and Vidale, 1991) or by a simple inverse distance approximation. In practice, we find that they can be ignored.

4) The slowness model is updated and these steps are iteratively repeated until convergence. Quintus-Bosz (1992) and Quintus-Bosz and Schuster (paper in preparation) have successfully tested the effectiveness of this scheme.

Numerical examples

We will now present some numerical examples that illustrate how the WET method accounts for source bandwidth and shadow zone effects in the data. Figure 2 depicts a cylinder velocity model with a velocity contrast of $2: 1$, and Figures $3 \mathrm{a}$ and $3 \mathrm{~b}$ depict the traveltime contours for a line source at $\mathbf{x}_{s}$ and $\mathbf{x}_{r}$, respectively. These traveltimes were computed by a finite-difference solution to the eikonal equation (Qin et al., 1992). In this case, $\tau_{x r}$ and $\tau_{x s}$ represent common source point (CSP) gathers of traveltimes. In the following examples, the 2-D equivalent of equation (1 lc) was used to compute wavepaths (Quintus-Bosz, 1992; QuintusBosz and Schuster, paper in preparation); i.e., the 2-D rather than the 3-D asymptotic Green's function was used in equation (8) to derive the asymptotic gradient term.

\section{Calculations of the gradient function}

Some wavepath functions are now calculated for a lowvelocity cylinder model. The argument of the source function in equation $\left(1 \mathrm{lc}\right.$ ) is calculated by subtracting $\tau_{r s}$ (for a fixed $r$ and s) from the Figure (3a) and Figure (3b) summed traveltime values to yield the traveltime contours in Figure (3c). Note that the two zero traveltime contours correspond to the two raypaths associated with the first arrival so that some multipathing effects are partially taken into account. This compares favorably to ray-tracing methods that usually compute only a single raypath per sourcereceiver pair. Other contours correspond to the aplanatic surfaces of nonzero positive traveltimes.

The traveltimes contoured in Figure $3 \mathrm{c}$ are used in the argument of the weighting function in equation $(1 \mathrm{lc})$ to give a plot of the gradient function in Figure 3d. The weighting function is taken to be a Ricker wavelet where the peak frequency is $100 \mathrm{~Hz}$; in addition, the geometrical spreading terms are neglected. This figure shows that the slowness model is mostly updated along and near the two wavepaths that are tangent to the top and bottom of the cylinder. Unlike the corresponding raypath, the wavepath penetrates into the low-velocity interior of the cylinder.

Band-limited source effects

The wavepath width decreases as the peak Ricker source frequency increases [i.e., as $\omega_{0}$ increases in equation $(1 \mathrm{lc})$ ]. This is illustrated in Figure 3e where the peak frequency of the source spectrum is four times that in Figure 3d. Equivalently, as the period of the source wavelet increases, a larger region of the model can contribute scattered energy to the observed first-arrival wavelet. This larger region is encompassed by the support of the enlarged wavepath.

\section{Shadow effects}

Unlike shooting ray-tracing algorithms, finite-difference solutions to the eikonal equation can compute first-arrival times associated with some diffraction first arrivals (Vidale, 1988). In addition, the geometrical spreading terms in equation (11) are used to weight the traveltime residuals. Traveltime residuals associated with weak amplitude arrivals have less weight than those associated with large amplitude arrivals. This is an automatic means for discriminating against arrivals with questionable signal to noise ratios.

Reconstruction of a cylinder model by WET

Inversion with band-limited fat rays or wavepaths produces smooth tomograms because the traveltime residuals

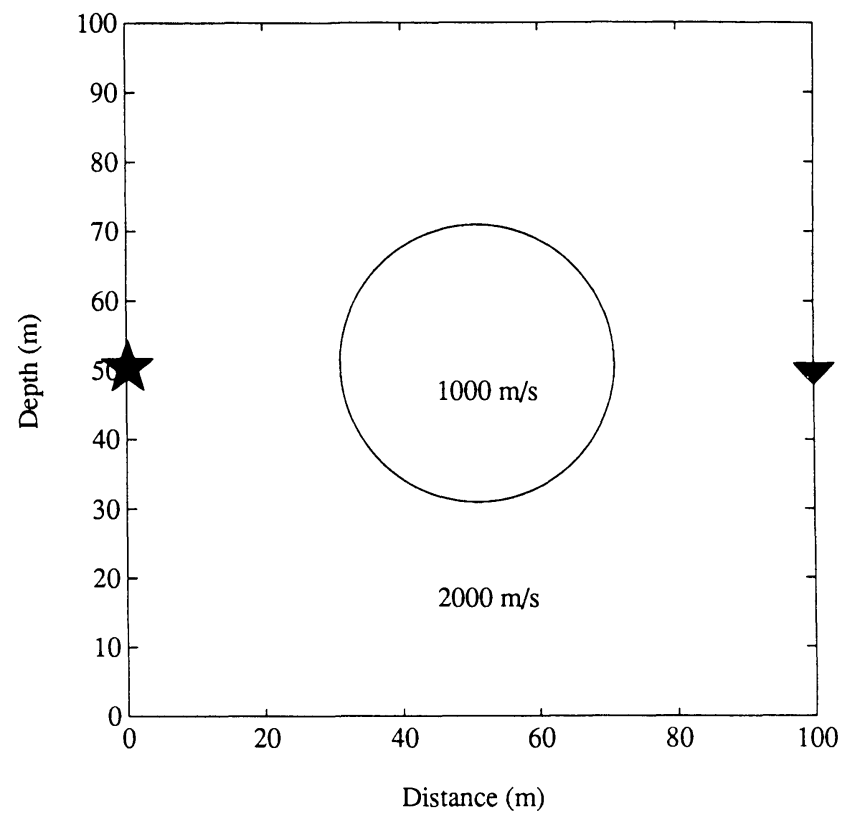

FIG. 2. Low-velocity cylinder model, with the interior (exterior) velocity equal to $1 \mathrm{~km} / \mathrm{s}(2 \mathrm{~km} / \mathrm{s})$. 
a)

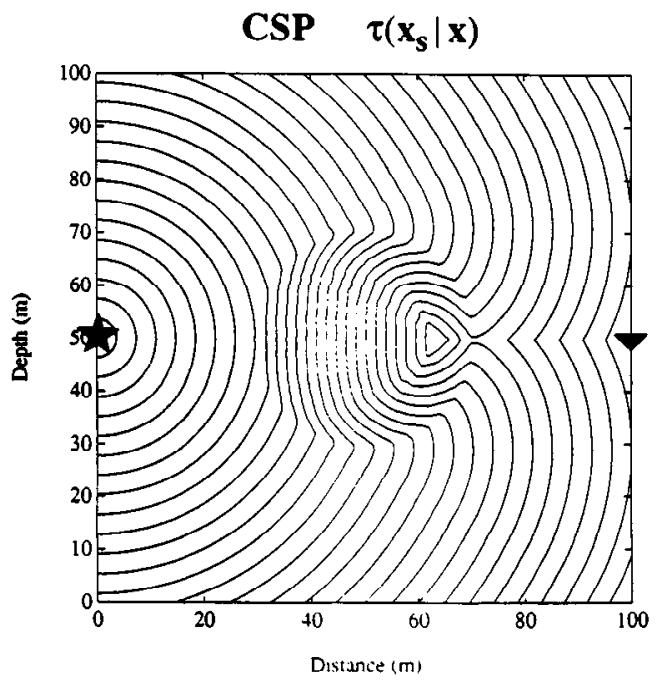

b)

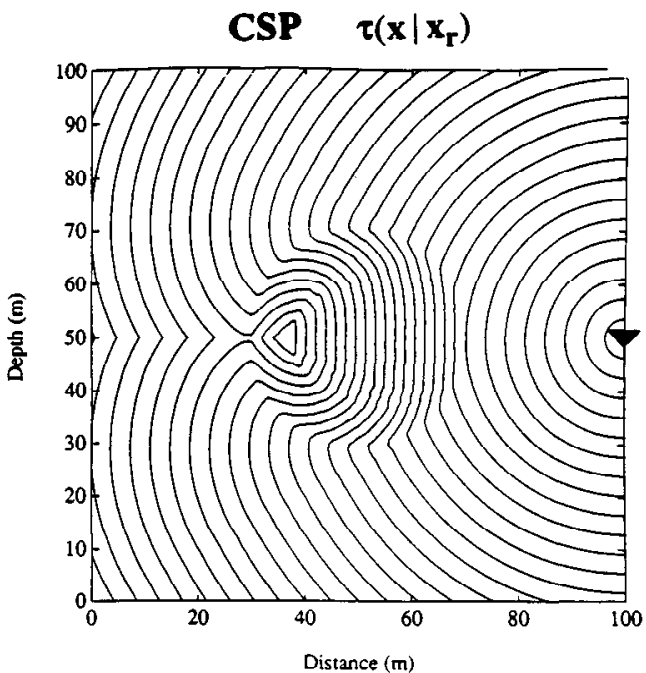

c)

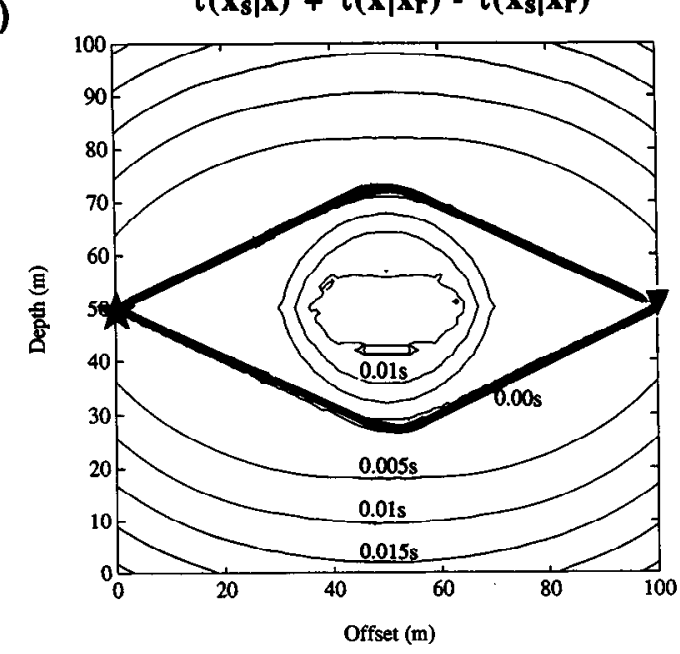

d) $0 \mathrm{~m}$

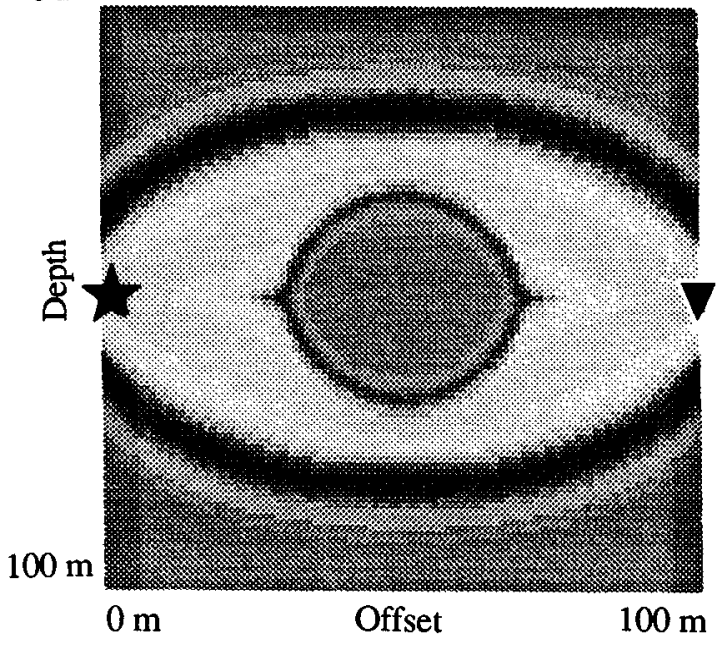

e)

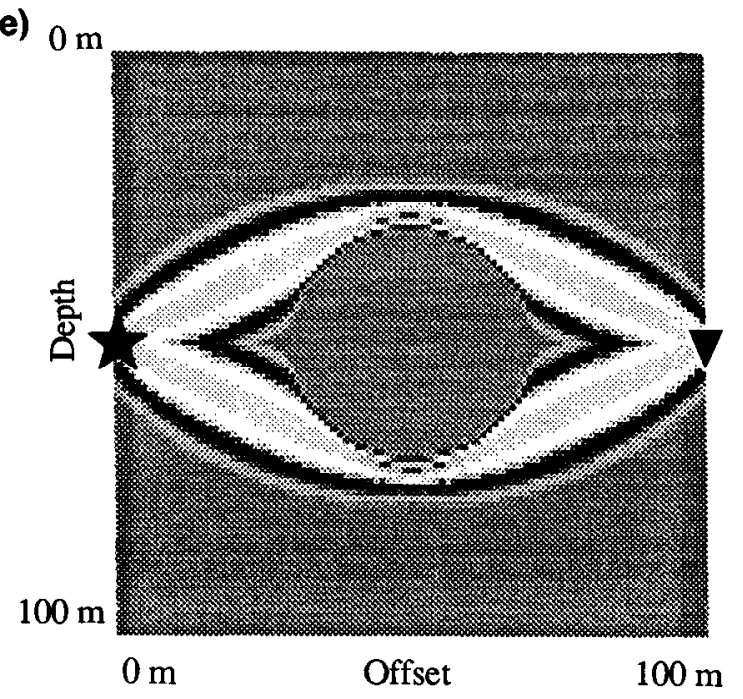

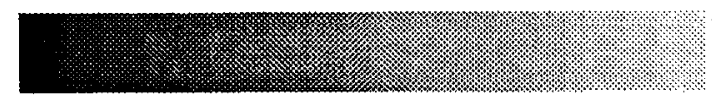

$-70 \mathrm{~m} / \mathrm{sec}$

Velocity update magnitude

FIG. 3. (a) First-arrival traveltime contours for a source located at the star position on the left side of Figure 2. (b) First-arrival traveltime contours for a source located at the receiver position on right side of Figure 2 . (c) Traveltime contours computed by adding the traveltimes in Figures $3 \mathrm{a}$ and $3 \mathrm{~b}$ and subtracting $\tau_{r s}$. The dark solid line indicates the zero time contour, which is coincident with the first-arrival raypath. (d) Gradient function in equation (1 lc) computed from the traveltimes in Figure $3 \mathrm{c}$, with a Ricker wavelet function having a $100 \mathrm{~Hz}$ peak frequency; also the amplitude terms have been neglected. (e) Same as Figure $3 \mathrm{~d}$ except the peak frequency has been quadrupled from $100 \mathrm{~Hz}$ to $400 \mathrm{~Hz}$. 
are back projected over a wide swath of pixels in the slowness model. That is, the WET method updates the slowness field as a continuous function of the spatial coordinates. This compares favorably to undamped RT methods that update the slowness model unevenly because of the thin nature of the rays. For example, slowness pixels sampled by many rays can experience a large update due to the effect of each ray's additive contribution. The slowness of these pixels may be changed significantly, while that of unvisited pixels is not updated at all. Therefore, RT methods require smoothing to damp the resulting artifacts in the tomograms.

Two examples are used to show the smoothing effects of wavepaths: one with a dense data set, and another with a sparse data set. The model is a cylindrical high-velocity $(3 \mathrm{~km} / \mathrm{s})$ zone six wavelengths in diameter, embedded in a homogeneous background $(2.5 \mathrm{~km} / \mathrm{s})$ ten wavelengths wide. The eikonal equation solver of Qin et al. (1992) provided 5041 "observed" traveltimes (71 sources and 71 receivers) for the dense data set, and 121 traveltimes (11 sources and 11 receivers) for the sparse data set. There are $71 \times 141=$ 10011 slowness pixels in this model.

Dense data case.-Figure 4 shows the WET (4a), WT (4b), and smoothed RT (4c) velocity tomograms associated with the dense data set. The RT method implements smoothing by a constraint that minimizes the roughness of the slowness update every iteration (Sun and Schuster, 1992). The three tomograms

a)

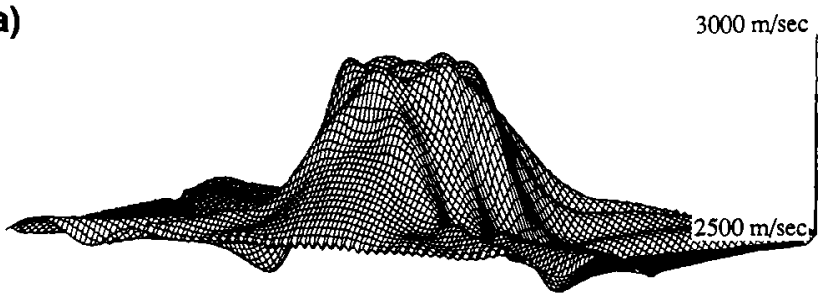

b)

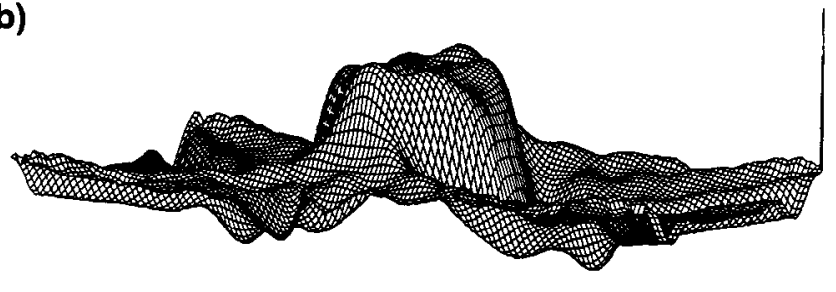

c)

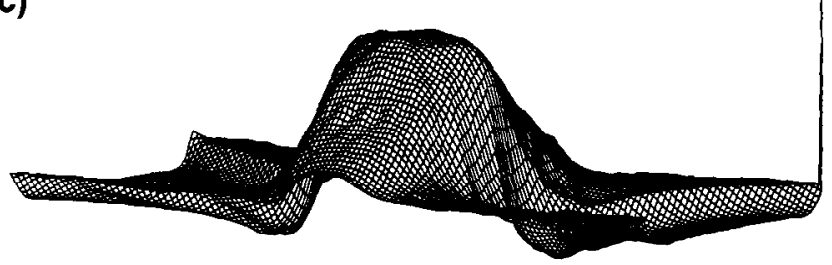

FIG. 4. Dense data set for a cylindrical model. (a) WET tomogram (400 Hz Ricker source wavelet); (b) WT tomogram (200 Hz Ricker source wavelet); (c) Smoothed-RT tomogram. in Figure 4 are qualitatively successful in imaging the highvelocity zone. For a dense data set the RT and WET methods perform equally well. Note that the WT tomogram underestimates the high velocity zone. This results from the lower resolution of a low-frequency (200 Hz peak frequency) source wavelet used for the WT inversion. Higher frequency wavelets required excessive computational effort, and were not used.

Sparse data case.-Figure 5 shows the WET (5a), WT (5b), and unsmoothed RT (5c) velocity tomograms associated with the sparse data set. The WET and WT tomograms are much smoother and retain the essential features of the actual model. In contrast, the unsmoothed RT tomogram in Figure $5 c$ is very noisy and conveys little information. Unconstrained RT leads to tomograms with high-wavenumber variations in slowness. Without adequate smoothing, "spikes" can pollute the final RT tomogram as shown in Figure 5d. The WET method, however, fits the data using slowness models with a spatial frequency content that is consistent with the source wavelet's finite bandwidth. In other words, the wavepaths act as physically consistent low pass or anti-alias filters to the reconstructed slowness model.
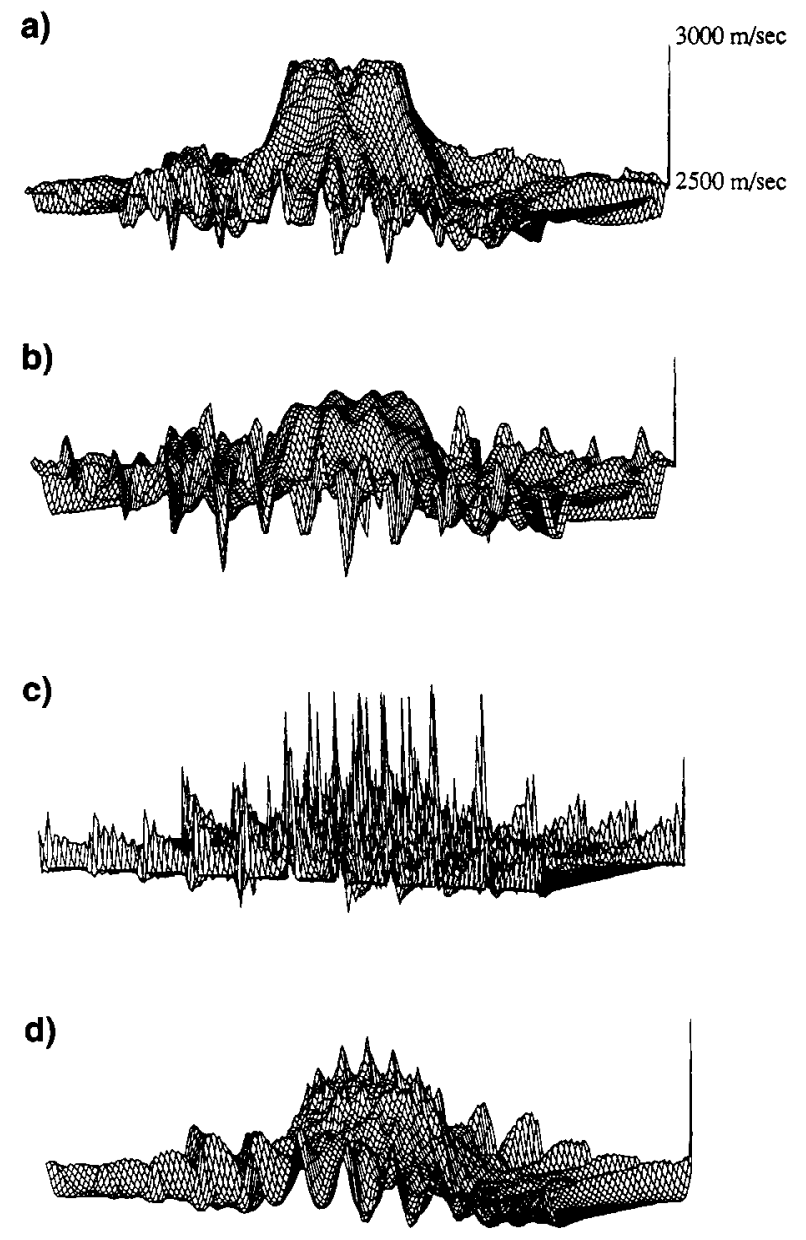

FIG. 5. Sparse data set for a cylindrical model. (a) WET tomogram (400 Hz Ricker source wavelet); (b) WT tomogram (200 Hz Ricker source wavelet); (c) RT tomogram; (d) Smoothed-RT tomogram. 


\section{SUMMARY}

A traveltime back-projection formula in equation (8) is given which reduces, as special cases of the weighting factor $\widetilde{R}_{r s}(\omega)$, to the asymptotic back-projection formula for the thin ray RT, fat ray RT, WET, WR, and WT methods. The chief effect of this weighting factor is to smooth the gradient (or reconstructed slowness field) in a way that is physically consistent with the source spectrum and the path of propagation. This is more physically appealing than ad hoc smoothing methods. Table 1 summarizes these weighting factors and shows that:

1) Traveltime residuals are back projected along wavepaths defined by the source wavelet (fat ray RT), the weighted first derivative of the sinc function (WR), the weighted third derivative of the source wavelet (WET), and the weighted third derivative of the actual first arrival waveform (WT).

2) The preconditioning term of Beydoun and Mendes (1989) suggests that the geometrical spreading factors may not be important for the asymptotic WET, WT, or WR inversion methods.

3) The different order of derivatives suggests that the asymptotic WR and fat RT methods provide low-pass tomograms relative to the WET and asymptotic WT tomograms.

4) The asymptotic WT method uses the observed firstarrival waveform to define the back-projection operator. This is a compeling feature since it partially takes into account the scattering effects in the data.

The WET algorithm is, in principle, almost as effective as WT inversion yet is an order of magnitude faster; only solutions to the transport and eikonal equation are involved. Unlike some ray-tracing tomography algorithms (1) WET partially accounts for source bandwidth and shadow effects in the data, and (2) its inherent smoothing acts as a physically consistent low-pass or anti-aliasing filter to the reconstructed model. The penalty, however, is that WET ignores scattering effects by linearizing phase with respect to frequency. The effectiveness of a multigrid WET method applied to real and synthetic cross-well data is shown.

So which asymptotic tomography method is best? In principle, the WT method should be very desirable because it accounts for waveform shape, scattering and real data effects in defining the back-projection paths. WET should be a good choice because it accounts for the source wavelet shape and is consistent with minimization of the $\ell_{2}$ norm of the traveltime residuals. Asymptotic WR and fat ray RT methods should be the last choice because they both provide only low-pass versions of the WT or WET tomograms. However, our limited experience with synthetic data suggests (Quintus-Bosz and Schuster, paper in preparation) that RT with optimal smoothing performs about as well as the WET or WT methods. If the velocity contrasts become too large (greater than two or three to one) then ray-traced traveltimes should be replaced by finite-difference solutions to the eikonal equation.
Future research might explore the application of a WETlike algorithm to the inversion of refraction traveltimes, attenuation data, and anisotropic traveltimes.

\section{ACKNOWLEDGMENTS}

The authors are very grateful for the financial support from the 1990 sponsors of the University of Utah Seismic tomography consortium: Amerada Hess, Amoco, Arco, British Petroleum, Chevron, Conoco, Exxon, Gas Research Institute, Francais du Petrole, Japon, Marathon, Mobil, Oryx, Oyo, Phillips, Texaco, Unocal, and Western Geophysical. We would like to thank Fuhao Qin for helping with the calculations and figures, Yi Luo for useful discussions, and Yonghe Sun for his review and for many useful discussions.

\section{REFERENCES}

Beydoun, W., and Mendes, M., 1989, Elastic ray-Born $\ell_{2}$ migration/inversion: Geophys. J., 97, 151-160.

Cerveny, V., and Soares, J, 1992, Fresnel volume ray tracing: Geophysics, 57, 902-915.

Gersztenkorn, A., and Scales, J., 1988, Smoothing seismic tomograms with alpha-trimmed means: Geophys. J., 92, 67-72.

Harlan, W., 1990, Tomographic estimation of shear velocities from shallow cross-well seismic data: 60th Ann. Internat. Mtg., Soc. Expl. Geophys., Expanded Abstracts, 86-89.

Keller, J. B., 1969, Accuracy and validity of the Born and Rytov approximations: J. Opt. Soc. Am., 59, 1003-1004.

Luo, Y., 1991, Calculation of wavepaths in the time domain: 61st Ann. Internat. Mtg., Soc. Expl. Geophys., Expanded Abstracts, $1509-1512$

Luo, Y., and Schuster, G. T., 1990, Wave equation traveltime inversion: 60th Ann. Internat. Mtg., Soc. Expl. Geophys. Expanded Abstracts, 1207-1210. 645-653. 1991a, Wave equation traveltime inversion: Geophysics, 56,

1991 b. Wave equation inversion of skeletalized geophysical data: Geophys. J. 105:2, 289-294.

Matsuoka, T., and Ezaka, T., 1992, Ray tracing using reciprocity: Geophysics, 57, 326-333.

Meyerholtz, K., Pavlis, G., and Szpakowski, S., 1989, Convolutional quelling in seismic tomography: Geophysics, 54, 570-580.

Miller, D., Oristaglio, M., and Beylkin, G., 1987, A new slant on seismic imaging: Migration and integral geometry: Geophysics, 52, 943-964

Pratt, G., and Worthington, M., 1990, Inverse theory applied to multisource crosshole tomography. Part 1: Acoustic wave equation method: Geophys. Prosp. 38, 287-310.

Pusey, L., and Vidale, J., 1991, Accurate finite-difference calculation of WKBJ traveltimes and amplitudes: 61st Ann. Internat. Mtg., Soc. Expl. Geophys., Expanded Abstracts, 1513-1516.

Qin, F., Luo, Y., Olsen, K., Cai, W., and Schuster, G., 1992, Finite-difference solution of the eikonal equation: Geophysics, 57, 478-487.

Quintus-Bosz, A., 1992, Wavepath eikonal equation traveltime inversion: MS thesis, University of Utah.

Schuster, G. T., 1991, Wave-equation phase inversion in the frequency domain: 61st Ann. Internat. Mtg., Soc. Expl. Geophys., Expanded Abstracts, 909-912.

Sun, Y., and Schuster, G. T., 1992, Hierarchic optimizations for smoothing and cooperative inversion: 62nd Ann. Internat. Mtg., Soc. Expl. Geophys. Expanded Abstract, 745-748.

Tarantola, A., 1987, Inverse problem theory: Elsevier Science Publ.

Vidale, J., 1988, Finite-difference calculation of traveltimes: Bull. Seis. Soc. Am. 78, no. 6, 2062-2076.

Woodward, M., 1989, Wave equation tomography: Ph.D. Dissertation, Stanford University.

1992, Wave-equation tomography: Geophysics, 57, 15-26.

Woodward, M., and Rocca, F., 1988, Wave-equation tomography: 58th Ann. Internat. Mtg., Soc. Expl. Geophys., Expanded Abstracts, 1232-1235. 


\section{APPENDIX \\ SPECIAL CASES OF WET ANALOGIES TO WT AND WR GRADIENTS}

This Appendix shows how special cases of the weighting factor $\widetilde{R}_{r s}(\omega)$ in equation (8) reduce to the asymptotic WT and Woodward and Rocca gradients.

\section{WT asymptotic gradient}

For a single source location $\mathbf{x}_{s}$, a single receiver location $X_{r}$, the WT gradient of the traveltime misfit function in equation (8a) of Luo and Schuster (1991a) can be rewritten as

$$
\begin{aligned}
\frac{\partial \hat{\varepsilon}}{\partial s(\mathbf{x})}= & \frac{-2 s(\mathbf{x}) \Delta \tau}{E} \int_{-\infty}^{\infty} d t \dot{p}\left(\mathbf{x}_{r}, t-\Delta \tau \mid \mathbf{x}_{s}, 0\right)_{o b s} \\
& \times p^{\prime}\left(\mathbf{x}, \mathbf{x}_{r}, \mathbf{x}_{s}, t\right),
\end{aligned}
$$

where

$p^{\prime}\left(\mathbf{x}, \mathbf{x}_{r}, \mathbf{x}_{s}, t\right)=-\dot{g}\left(\mathbf{x}, t \mid \mathbf{x}_{r}, 0\right) * \dot{p}\left(\mathbf{x}_{r}, t \mid \mathbf{x}_{s}, 0\right)_{c a l}$,

and $E$ is a scaling factor defined by

$$
E=-\int d t \ddot{p}\left(\mathbf{x}_{r}, t-\Delta \tau \mid \mathbf{x}_{s}, 0\right)_{o b s} p\left(\mathbf{x}_{r}, t \mid \mathbf{x}_{s}, 0\right)_{c a l} .
$$

Here the velocity gradient in Luo and Schuster (1991a) is converted to a slowness gradient, $\hat{\varepsilon}$ is the sum of the squared traveltime residuals, and AT is the difference between the peak amplitude traveltimes of the observed $\tau_{r s}^{o b s}$ and calculated $\tau_{r s}$ first arrivals, i.e.,

$$
\Delta \tau=\tau_{r s}-\tau \underset{r s}{o b s} .
$$

In equation (A-2), a dot indicates time differentiation, * denotes convolution in time, and $p\left(\mathbf{x}, t \mid \mathbf{x}_{s}, 0\right)_{c a l}$ is the calculated space-time pressure field for the slowness distribution $\mathrm{s}(\mathrm{x})$.

For high frequencies the asymptotic inhomogeneous Green's function is

$$
\begin{aligned}
p\left(\mathbf{x}, t \mid \mathbf{x}^{\prime}, t^{\prime}\right)_{c a l} & =g\left(\mathbf{x}, t \mid \mathbf{x}^{\prime}, \mathbf{t}^{\prime}\right) \\
& =\frac{\delta\left(\left[t-t^{\prime}\right]-\tau\left(\mathbf{x}, \mathbf{x}^{\prime}\right)\right)}{A_{x x^{\prime}}},
\end{aligned}
$$

where $\tau\left(\mathbf{x}, x^{\prime}\right)$ is the solution to the eikonal equation for a source (receiver) at $\mathrm{x}^{\prime}(\mathrm{x})$ and $A_{x x^{\prime}}$ is the corresponding geometrical spreading term. Substituting equation (A-5) into equation (A-3) gives

$$
E=\frac{-\ddot{p}\left(\mathbf{x}_{r}, \tau_{r s}-\Delta \tau \mid \mathbf{x}_{s}, \mathbf{0}\right)_{o b s}}{A_{r s}}=\frac{-\ddot{p}\left(\mathbf{x}_{r}, \tau_{r s}^{o b s} \mid \mathbf{x}_{s}, 0\right)_{o b s}}{A_{r s}}
$$

where the last expression follows by definition of $\Delta \tau$. Substituting equation (A-5) into equation (A-2) gives

$$
p^{\prime}\left(\mathbf{x}, \mathbf{x}_{r}, \mathbf{x}_{s}, t\right)=\frac{\ddot{\delta}\left(t-\tau_{x s}-\tau_{x r}\right)}{A_{x r} A_{x s}},
$$

and substituting equations (A-7) and (A-6) into equation (A-1) yields the asymptotic form of the WT gradient

$$
\begin{aligned}
& \frac{\partial \hat{\varepsilon}}{\partial s(\mathbf{x})} \\
& =\frac{2 s(\mathbf{x}) \Delta \tau A_{r s} \ddot{p}\left(\mathbf{x}_{r}, \tau_{x s}+\tau_{x r}-\tau_{r s}+\tau_{r s}^{o b s} \mid \mathbf{x}_{s}, 0\right)_{o b s}}{A_{x s} A_{x r}} .
\end{aligned}
$$

Alternatively, setting the weighting factor in the general asymptotic gradient in equation (8) to be

$$
\tilde{R}_{r s}(\omega)=\frac{2 \operatorname{Im}\left(i \tilde{p}\left(\mathbf{x}_{r}, \omega \mid \mathbf{x}_{s}, 0\right)_{o b s} e^{i \omega \tau_{r s} h s}\right)}{\ddot{p}\left(\mathbf{x}_{r}, \tau_{r s}^{o b s} \mid \mathbf{x}_{s}, 0\right)_{o b s}}
$$

leads to the WT gradient in equation (A-8). This follows from the fact that if $\mathrm{f}(\mathrm{t})$ is a real causal function then $\mathrm{f}(\mathrm{t})=$ $\int \widetilde{f}(\omega) e^{i \omega t} d o=-2 \int \operatorname{Im}(\widetilde{f}(\omega)) \sin (w t) d o$. Note that the WT gradient in equation (A-8) reduces to the WET gradient of equation (1 lc) if $p\left(\mathbf{x}_{r}, t \mid \mathbf{x}_{s}, 0\right)$ is set equal to the zero phase Ricker wavelet $W_{r s}(t)$. This varifies that the gradient in equation (11c) is nothing more than $\Delta \tau \partial \tau / \partial s$.

From equation (A-8) we see that the WT gradient uses the actual first arrival windowed from the observed seismogram, not just the source wavelet. In part, this takes into account the effects of scattering and source radiation pattern in the data. The above formula suggests that an efficient implementation of WT might use the synthetic traveltimes computed by finite-difference solutions to the eikonal equation, rather than the wave equation.

Woodward-Rocca asymptotic gradient

For a single source location $\mathbf{x}_{s}$, a single receiver location $X_{r}$, the multifrequency gradient (for inverting normalized velocity Avlv) in equation (18) of Woodward (1992) is given as

$$
\begin{aligned}
\gamma(\mathbf{x}) & \stackrel{2 \Delta \tau s(\mathbf{x})^{2}}{\Delta \omega} \int \omega \operatorname{Im}\left(\frac{\tilde{g}\left(\mathbf{x}, \mathbf{x}_{r}\right) \tilde{g}\left(\mathbf{x}, \mathbf{x}_{s}\right)}{\tilde{g}\left(\mathbf{x}_{r}, \mathbf{x}_{s}\right)}\right) d \omega \\
& =\frac{2 \Delta \tau s(\mathbf{x})^{2}\left|g\left(\mathbf{x}, \mathbf{x}_{r}\right)\right|\left|g\left(\mathbf{x}, \mathbf{x}_{s}\right)\right|}{\Delta \omega} \int \omega \sin [\phi] d \omega
\end{aligned}
$$

where $\phi$ is the total phase of the concatenated 3-D Helmholtz Green's functions, $\Delta \omega$ is the bandwidth of integration, and their notation for Green's function is now used. If the asymptotic Green's function in equation (7) is substituted into equation (A-10) and the limits of integration are taken to be from $-\omega_{0}$ to $+\omega_{0}$, then

$$
\begin{aligned}
\gamma(\mathbf{x}) & =\frac{s(\mathbf{x})^{2}}{\omega_{0}} \frac{A_{r s} \mathrm{AT}}{A_{x s} A_{x r}} \int_{-\omega_{0}}^{\omega_{0}} \omega \sin \left[\omega\left(\tau_{x s}+\tau_{x r}-\tau_{r s}\right)\right] d \omega, \\
& =\frac{-2 s(\mathbf{x})^{2} A_{r s} \Delta \tau}{A_{x s} A_{x r}} \operatorname{sinc}\left[\omega_{0}\left(\tau_{x s}+\tau_{x r}-\tau_{r s}\right) / \pi\right]^{\prime} .
\end{aligned}
$$


Dividing the above by $-s(\mathbf{x})$ converts from Woodward's normalized velocity perturbation $\Delta v / v$ parameter to the slowness parameter As to yield

$$
\gamma(\mathbf{x})=\frac{2 \Delta \tau s(\mathbf{x}) A_{r s}}{A_{x s} A_{x r}} \operatorname{sinc}\left[\omega_{0}\left(\tau_{x s}+\tau_{x r}-\tau_{r s}\right) / \pi\right]^{\prime} .
$$

Alternatively, this gradient could have been derived by setting $R,,(O)=-1 / \omega^{2}$. The interpretation of equation (A-1 1) is that the traveltime residuals are back-projected along the wavepaths defined by the first derivative of the sinc function with the same bandwidth of the source wavelet. This takes into account source bandwidth but not the source wavelet's shape. 